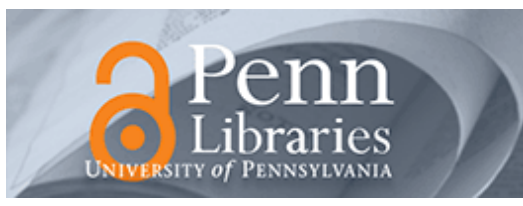

University of Pennsylvania ScholarlyCommons

Finance Papers

Wharton Faculty Research

1983

\title{
On the Econometric Testing of Rationality-Market Efficiency
}

Andrew B. Abel

University of Pennsylvania

Frederic S. Mishkin

Follow this and additional works at: http://repository.upenn.edu/fnce_papers

Part of the Econometrics Commons, Finance Commons, and the Finance and Financial Management Commons

\section{Recommended Citation}

Abel, A. B., \& Mishkin, F. S. (1983). On the Econometric Testing of Rationality-Market Efficiency. The Review of Economics and Statistics, 65 (2), 318-323. http://dx.doi.org/10.2307/1924498

At the time of publication, author Andrew B. Abel was affiliated with Harvard University. Currently, he is a faculty member at the Wharton School at the University of Pennsylvania.

This paper is posted at ScholarlyCommons. http://repository.upenn.edu/fnce_papers/249

For more information, please contact repository@pobox.upenn.edu. 


\title{
On the Econometric Testing of Rationality-Market Efficiency
}

\author{
Abstract \\ No abstract provided. \\ Disciplines \\ Econometrics | Finance | Finance and Financial Management
}

\section{Comments}

At the time of publication, author Andrew B. Abel was affiliated with Harvard University. Currently, he is a faculty member at the Wharton School at the University of Pennsylvania. 


\title{
NOTES
}

\section{ON THE ECONOMETRIC TESTING OF RATIONALITY-MARKET EFFICIENCY}

\author{
Andrew B. Abel and Frederic S. Mishkin*
}

\section{Introduction}

Procedures for testing the rational expectations hypothesis deserve careful study because rationality of expectations has such important implications for macroeconomic modeling and policy analysis. ' Rationality of expectations generally imposes cross-equation restrictions. ${ }^{2}$ In this paper we discuss the implementation and analyze the econometric properties of a particular test of such restrictions. This test has been used in recent empirical studies of bond market behavior. ${ }^{3}$ Since the test focuses on the distinction between anticipated and unanticipated movements in variables (as in Barro (1977, 1978)), it is applicable to many macroeconomic issues. Indeed, the results of this paper are useful in further work (Abel and Mishkin (1983)) that clarifies the relations among tests of (1) rationality and market efficiency, (2) the short-run neutrality of anticipated policy and (3) Granger (1969) causality in macroeconometric models.

Two important questions about the test of cross-equation restrictions arise naturally. First, under what conditions will the test lead to correct inference about the rationality of expectations? Second, what is the relation of this cross-equation test to the more common singleequation test of market efficiency frequently used in the literature? The answers to these questions are provided by the theorem in section II which states the asymptotic equivalence of the cross-equation test with the more common test of market efficiency. Also in section II we discuss identification and demonstrate that we can test

Received for publication November 12, 1981. Revision accepted for publication August 20, 1982.

*Harvard University and National Bureau of Economic Research, and University of Chicago and National Bureau of Economic Research, respectively.

We thank John Abowd, Dennis Carlton, Robert Hall, John Huizinga, Bennet McCallum, Robert Shiller, Gary Skoog, Mark Watson, Arnold Zellner, two anonymous referees and the participants in the Econometrics and Statistics Colloquium at the University of Chicago and the Macroeconomics Seminar at the NBER Summer Institute for their helpful comments and suggestions. Research support from the National Science Foundation is gratefully acknowledged. The usual disclaimer applies.

1 See, for example, Sargent and Wallace (1975), Lucas (1976), Poole (1976) and Mishkin (1978).

${ }^{2}$ See Sargent $(1978,1979)$, for example.

${ }^{3}$ See Jones and Roley (1981), Mishkin (1981a, b, 1982) and Plosser (1982). for rationality of expectations even if some parameters are not identified. Finally, we present an empirical example in section III and concluding remarks in section IV.

\section{Tests of Rationality and Market Efficiency}

Let $R_{t}$ denote the return from holding a particular security from the end of period $t-1$ to the end of period $t$, and let $\phi_{t-1}$ denote the set of information available at the end of period $t-1$. Rationality of expectations, or equivalently, capital market efficiency, implies that the subjective expectation of $R_{t}$ assessed by the market is equal to the objective expectation conditional on past available information, $\phi_{t-1}$ :

$$
E_{m}\left(R_{t} \mid \phi_{t-1}\right)=E\left(R_{t} \mid \phi_{t-1}\right)
$$

where $E_{m}\left(R_{t} \mid \phi_{t-1}\right)$ is the subjective expectation assessed by the market. A slightly weaker condition ${ }^{4}$ is used in empirical applications,

$$
E\left(y_{t} \mid \phi_{t-1}\right)=0
$$

where

$$
y_{t} \equiv R_{t}-E_{m}\left(R_{t} \mid \phi_{t-1}\right) \text {. }
$$

In order to give (2) empirical content, we must specify a model of market equilibrium which determines $E_{m}\left(R_{t} \mid \phi_{t-1}\right)$. The reader is referred to section III for an example and to Fama (1976) for further discussion of various models of market equilibrium used to determine $E_{m}\left(R_{t} \mid \phi_{t-1}\right)$ in empirical work. Tests of (2) are tests of the joint hypothesis of market efficiency (rational expectations) and that the model of market equilibrium is correctly specified in computing $y_{t}$. For expositional convenience we refer to this joint hypothesis as "the efficient markets model."

Since equation (2) implies that $y_{t}$ should be uncorrelated with any available information in $\phi_{t-1}$, market efficiency is commonly tested by testing the null hypothesis that $\alpha=0$ in the equation below:

$$
y_{t}=Z_{t-1} \alpha+\mu_{t}
$$

\footnotetext{
${ }^{4}$ To see that (2) is weaker than (1), consider the case in which the market's subjective expectation is equal to the objective expectation plus an unforecastable observation error, that is, $E_{m}\left(R_{t} \mid \phi_{t-1}\right)=E\left(R_{t} \mid \phi_{t-1}\right)+q_{t}$ where $E\left(q_{t} \mid \phi_{t-1}\right)=0$. In this case condition (2) is satisfied but unless $q_{t} \equiv 0$, condition (1) is violated.
} 
where $Z_{t-1}$ is an $h$-element row vector of information contained in $\phi_{t-1}, \alpha$ is an $h \times 1$ vector of coefficients, and $\mu_{t}$ is a disturbance where $E\left(\mu_{t} \mid \phi_{t-1}\right)$ is assumed to equal zero.

If the market has rational expectations about $X$ so that $E\left(\left(X_{t}-E_{m}\left(X_{t} \mid \phi_{t-1}\right)\right) \mid \phi_{t-1}\right)=0$, then a characterization of the efficient markets model which satisfies (2) is

$$
y_{t}=\left(X_{t}-E_{m}\left(X_{t} \mid \phi_{t-1}\right)\right) \beta+\epsilon_{t}^{*}
$$

where $\epsilon_{t}^{*}$ is a scalar disturbance with the property $E\left(\epsilon_{t}^{*} \mid \phi_{t-1}\right)=0, X_{t}$ is a $k$-element row vector containing variables relevant to the pricing of the security at time $t$, and $\beta$ is a $k \times 1$ vector of coefficients. As is evident in (4), only unanticipated changes in $X_{t}$ can be correlated with $y_{t}$.

Suppose that the linear model for the $k$ variables in $X$ can be written as

$$
X_{t}=Z_{t-1} \gamma+u_{t}
$$

where $\gamma$ is a $h \times k$ matrix of coefficients and $u_{t}$ is a $k$-element row vector of disturbances. Suppose, for the moment, that $E\left(u_{t} \mid \phi_{t-1}\right)=0$ so that $E\left(X_{t} \mid \phi_{t-1}\right)=$ $Z_{t-1} \gamma$. Then, provided that $E\left(\left(X_{t}-E_{m}\left(X_{t} \mid \phi_{t-1}\right)\right) \mid \phi_{t-1}\right)$ $=0$, equation (4) can be written as

$$
y_{t}=\left(X_{t}-Z_{t-1} \gamma^{*}\right) \beta+\epsilon_{t}
$$

where $\gamma=\gamma^{*}$, and $E\left(\epsilon_{t} \mid \phi_{t-1}\right)=0 .^{5}$

The system in (5) and (6) can be stacked into one regression system with $n(k+1)$ observations, and estimated by non-linear least squares. ${ }^{6}$ The cross-equation constraints implied by market efficiency, $\gamma=\gamma^{*}$, can then be tested with a likelihood ratio test. Two questions arise as to the econometric properties of this test. First, can this test be used for valid inference if $Z_{t-1}$ excludes variables relevant to forecasting the variables in $X_{t}$ ? Second, what is the relation of this test to the common test for market efficiency using equation (3)? The following theorem provides answers to these related questions.

THEOREM: The likelihood ratio ( $L R$ ) test of the null hypothesis $\gamma=\gamma^{*}$ in (5) and (6) is asymptotically equivalent to an F-test of the null hypothesis $\alpha=0$ in (3).

Proof: Observe that the system in (5) and (6) can be rewritten as

$$
\begin{aligned}
& X_{t}=Z_{t}, \gamma+u_{t} \\
& y_{t}=Z_{t}, 1 \theta+u_{t} \beta+\epsilon_{t}
\end{aligned}
$$

\footnotetext{
5 If $E\left(\left(X_{t}-E_{m}\left(X_{t} \mid \phi_{t-1}\right)\right) \mid \phi_{t}-1\right)=0$ (which corresponds to the weaker definition of rationality in (2)), then $E_{m}\left(X_{t} \mid \phi_{t-1}\right)=$ $Z_{t-1} \gamma+\epsilon_{t}^{* *}$ where $E\left(\epsilon_{t}^{* *} \mid \phi_{t-1}\right)=0$. Therefore $\epsilon_{t}=\epsilon_{t}^{*}-\epsilon_{t}^{* *} \beta$ and $E\left(\epsilon_{t} \mid \phi_{t}, 1\right)=0$.

${ }^{6}$ For a detailed description of the estimation procedure, see Mishkin (1983)
}

where $\theta=\left(\gamma-\gamma^{*}\right) \beta$. The null hypothesis $\gamma=\gamma^{*}$ will be true only if $\theta=0$. $^{7}$

The parameters $\gamma$ and $\theta$ can each be estimated using Zellner's seemingly unrelated regressions technique since the disturbances $u_{t}$ and $u_{t} \beta+\epsilon_{t}$ are each uncorrelated with $Z_{t-1}$. Although the disturbances are correlated across equations, Zellner's technique reduces to equation-by-equation ordinary least squares (OLS) since the right-hand-side variables are identical in all equations. Therefore, the estimate of $\boldsymbol{\theta}$ in (7) is numerically identical to the OLS estimate of $\alpha$ in (3). Thus, an LR test of $\gamma=\gamma^{*}$ is asymptotically equivalent to an $F$-test of $\alpha=0{ }^{8}$

We now turn to identification and estimation of the remaining parameters: $\beta$ and the covariance matrix of $u_{t}$ and $\epsilon_{t}$. Let

$$
\Sigma=\left[\begin{array}{cc}
\sigma_{u u} & \sigma_{u \epsilon} \\
\sigma_{u \epsilon}^{\prime} & \sigma_{\epsilon \epsilon}
\end{array}\right]
$$

be the contemporaneous covariance matrix of $u_{t}$ and $\epsilon_{t}$ where $\sigma_{u u}$ is a $k \times k$ matrix, $\sigma_{u \epsilon}$ is a $k \times 1$ vector and $\sigma_{\epsilon \epsilon}$ is a scalar. Therefore, the covariance matrix of the disturbances $u_{t}$ and $u_{t} \beta+\epsilon_{t}$ in (7) is

$$
\Omega=\left[\begin{array}{cc}
\sigma_{u u} & \sigma_{u u} \beta+\sigma_{u \epsilon} \\
\beta^{\prime} \sigma_{u u}+\sigma_{u \epsilon}^{\prime} & \beta^{\prime} \sigma_{u u} \beta+2 \beta^{\prime} \sigma_{u \epsilon}+\sigma_{\epsilon \epsilon}
\end{array}\right] .
$$

Let the sample contemporaneous covariance matrix of the residuals from the estimated system in (7) be

$$
S=\left[\begin{array}{cc}
S_{X X} & S_{X y} \\
S_{X y}^{\prime} & S_{y y}
\end{array}\right]
$$

where $S_{X X}$ is a $k \times k$ matrix, $S_{X y}$ is a $k \times 1$ vector and $S_{y y}$ is a scalar. Using $S$ as our estimate of $\Omega$ we obtain

$$
\begin{aligned}
S_{X X} & =\hat{\sigma}_{u u} \\
S_{X y} & =\hat{\sigma}_{u u} \hat{\beta}+\hat{\sigma}_{u \epsilon} \\
S_{y y} & =\hat{\beta}^{\prime} \hat{\sigma}_{u u} \hat{\beta}+2 \hat{\beta}^{\prime} \hat{\sigma}_{u \epsilon}+\hat{\sigma}_{\epsilon \epsilon}
\end{aligned}
$$

where a circumflex denotes the estimate of a parameter. The estimate of $\sigma_{u u}$ is obtained directly from (11). However, without further a priori restrictions, the parameters $\beta, \sigma_{u \epsilon}$ and $\sigma_{\epsilon \epsilon}$ are not identified. Equations (12) and (13) contain $2 k+1$ parameters to be estimated and only $k+1$ equations. Thus, $k$ additional restrictions are required for identification. For example,

\footnotetext{
${ }^{7}$ Note that $\theta=0$ does not imply that $\gamma=\gamma^{*}$ since $\beta$ could be equal to zero. However, if $\beta$ is equal to zero, the parameter $\gamma^{*}$ is not identified and hence $\gamma=\gamma^{*}$ is not testable. Also, in the case in which $k>1$ so that $\beta$ is not a scalar, $\theta$ could equal zero even if $\beta \neq 0$ and $\gamma \neq \gamma^{*}$. In this case, the test of $\theta=0$ could fail to detect a violation of $\gamma=\gamma^{*}$ even asymptotically. See section V of Abel and Mishkin (1983) for further discussion of this point.

8 The tests are only asymptotically equivalent because of differences in degrees of freedom. See footnote 12.
} 
if the covariance vector $\sigma_{u \epsilon}$ is known, then $\beta$ and $\sigma_{\epsilon \epsilon}$ can be estimated from (12) and (13).

The null hypothesis $\theta=0$ in (7) can be tested with a likelihood ratio test with $h$ degrees of freedom. An asymptotically equivalent alternative to the likelihood ratio test is a Wald test based on the statistic

$$
Q=\hat{\theta}^{\prime}[V(\hat{\theta})]^{-1} \hat{\theta}
$$

where $V(\hat{\theta})$ is the variance of $\hat{\theta}$. Under the null hypothesis, $Q$ is distributed asymptotically as chi squared with $h$ degrees of freedom. Since the estimate of $\theta$ can be obtained from an OLS regression of $y$ on $Z$, it can be easily shown that

$$
V(\hat{\theta})=\left(\beta^{\prime} \sigma_{u u} \beta+2 \beta^{\prime} \sigma_{u \epsilon}+\sigma_{\epsilon \epsilon}\right)\left(Z^{\prime} Z\right)^{-1} .
$$

Recall from (13) that $S_{y y}$ provides an estimate of the scalar $\beta^{\prime} \sigma_{u u} \beta+2 \beta^{\prime} \sigma_{u \epsilon}+\sigma_{\epsilon \epsilon} .{ }^{9}$ Therefore, we estimate $Q$ in (14) by

$$
Q=\hat{\theta}^{\prime} Z^{\prime} Z \hat{\theta} / S_{\psi \nu}
$$

Except for the adjustment for degrees of freedom, the test statistic in (16) is the same as the test statistic for the null hypothesis $\alpha=0$ in (3). ${ }^{10}$

There are cases in which we might be interested in the coefficient estimates of the efficient markets model in (7) in order to study the effects of unanticipated movements in $X$ on the variable $y$ or to explore the source of rejections of market efficiency (rationality). ${ }^{11}$ In order to estimate $\beta$ consistently, we need to impose some identifying restrictions on the covariance vector $\sigma_{u \epsilon}$. If we assume that $\sigma_{u \epsilon}=0$, then it is particularly easy to estimate the constrained version $(\theta=0)$ of the system in (7) by non-linear weighted least squares in which the residual sums of squares are weighted by the inverse of

\footnotetext{
${ }^{9}$ Observe that the unidentifiability of $\beta, \sigma_{u \epsilon}$ and $\sigma_{\epsilon \epsilon}$ does not prevent estimation of the scalar $v^{2}=\left(\beta^{\prime} \sigma_{u u} \beta+2 \beta^{\prime} \sigma_{u \epsilon}+\sigma_{\epsilon \epsilon}\right)$ which is used in the test for the significance of $\theta$.

${ }^{10}$ Using a standard regression package, $S_{y y}$ would be calculated as $\hat{e}^{\prime} \hat{e} /(n-h)$ rather than $\hat{e}^{\prime} \hat{e} / n$, where $\hat{e}$ is the vector of residuals $y-Z \hat{\theta}$. Of course, this difference disappears asymptotically. Note, however, that although $\hat{\theta}$ may be obtained from a regression of $y$ on $Z$ and $(X-Z \hat{\gamma})$, the test statistics on $\hat{\theta}$ using this regression are inconsistent because the residuals from this regression, denoted by $\hat{\epsilon}$, yield an inconsistent estimate of

$$
\beta^{\prime} \sigma_{u u} \beta+2 \beta^{\prime} \sigma_{u \epsilon}+\sigma_{\epsilon \epsilon} \text {. }
$$

Let

$$
\hat{\epsilon}=y-(X-Z \hat{\gamma}) \hat{\beta}-Z \hat{\theta}=y-\hat{u} \hat{\beta}-Z \hat{\theta}
$$

and recall that $\hat{e}=y-Z \hat{\theta}$. Therefore, $\hat{e}=\hat{\epsilon}+\hat{u} \hat{\beta}$ so that $\hat{e}^{\prime} \hat{e}=\hat{\epsilon}^{\prime} \hat{\epsilon}+\hat{\beta}^{\prime} \hat{u}^{\prime} \hat{u} \hat{\beta}$, since $\hat{\epsilon}^{\prime} \hat{u}=0$. Hence,

$$
\begin{aligned}
\operatorname{plim} \frac{1}{n} \hat{e}^{\prime} \hat{e} & =\operatorname{plim} \frac{1}{n}\left(\hat{\epsilon}^{\prime} \hat{\epsilon}-\hat{\beta}^{\prime} \hat{u}^{\prime} \hat{u} \hat{\beta}\right) \\
& =\beta^{\prime} \sigma_{u u} \beta+2 \beta^{\prime} \sigma_{u \epsilon}+\sigma_{\epsilon \epsilon}-\operatorname{plim} \frac{1}{n} \hat{\beta}^{\prime} \hat{u}^{\prime} \hat{u} \hat{\beta} .
\end{aligned}
$$

${ }^{11}$ See Mishkin (1983).
}

the contemporaneous covariance matrix $\Sigma$. An estimate of this covariance matrix can be obtained from the sample covariance matrix $S$ from the unconstrained system in (7). Observe that $S_{X v}=\hat{u}^{\prime} \hat{\epsilon}$ where $\hat{u}=X-Z \hat{\gamma}$ and $\hat{\epsilon}=y-Z \hat{\theta}-\hat{u} \hat{\beta}$ are the residual vectors from the unconstrained system. Since $\hat{\epsilon}$ is the residual vector from an ordinary least squares regression of $y$ on $\hat{u}$ and $Z, \hat{\epsilon}$ will be orthogonal to $\hat{u}$. Therefore, $S_{X y}=0$ and the estimated covariance matrix will be block diagonal. Furthermore, because all of the right-hand side variables in the first $k$ equations are identical, this procedure will lead to the same parameter estimates and test statistics that would be obtained by weighting the variables in equation $i$ by $1 /\left(S S R_{i}\right)^{1 / 2}$, where $S S R_{i}=$ the sum of squared residuals in equation $i$.

If $\sigma_{u \epsilon} \neq 0$, then the procedure described above will not produce consistent estimates of $\beta$. However, the calculated test statistic for the null hypothesis $\theta=0$ will continue to be appropriate in this case. ${ }^{12}$

Recall that market efficiency, as described by equation (2), implies that $y_{t}$ is uncorrelated with any linear combination of available past information. Therefore, the common test of market efficiency has the desirable property that, except for a chance, a rejection of the null hypothesis can only occur if the market is not efficient, regardless of what available information is included in $Z$. However, a failure to reject the null hypothesis, even asymptotically, does not rule out market inefficiency. ${ }^{13}$ Because we have shown that the common test is asymptotically equivalent to the test of the cross-equation constraints in (5) and (6), it follows that the cross-equation test can also be used for inference under quite general conditions. That is, for any choice of the available information in $Z_{t-1}$ and for any value of $\sigma_{u \epsilon}$, a

12 Since $S_{X y}$, will equal zero, the estimated variance-covariance matrix for $\hat{\theta}$ will be calculated by a non-linear least squares program as

$$
V(\hat{\theta})=\hat{\sigma}^{2}\left(\hat{\beta}^{\prime} \hat{u}^{\prime} \hat{u} \hat{\beta}+\hat{\epsilon}^{\prime} \hat{\epsilon}\right)\left(Z^{\prime} Z\right)^{-1}
$$

where $\hat{\sigma}^{2}$ is the square of the standard error of the non-linear regression and is estimated as the total sum of squares divided by the degrees of freedom for this regression. Because of the weighting of each equation by $1 / \sqrt{S S R_{i}}$, the total sum of squares of the non-linear regression equals the number of stacked equations, i.e., $k+1$, while the degrees of freedom equals $(k+1)(n-h)-k$. Thus $\hat{\sigma}^{2}=(k+1) /[(k+1)$ $(n-h)-k]$ and the test statistic will be $Q=\hat{\theta}^{\prime} Z^{\prime} Z \hat{\theta} / \omega$ where

$$
\omega=\left[\frac{(k+1)}{(k+1)(n-h)-k}\right]\left(\hat{\beta}^{\prime} \hat{u}^{\prime} \hat{u} \hat{\beta}+\hat{\epsilon}^{\prime} \hat{\epsilon}\right) .
$$

Since $\hat{\theta}$ will be the same as that estimated in a regression of $y$ on $Z$ and since $\hat{\beta}^{\prime} \hat{u}^{\prime} \hat{u} \hat{\beta}+\hat{\epsilon}^{\prime} \hat{\epsilon}=(y-Z \hat{\theta})^{\prime}(y-Z \hat{\theta})$, the test statistic $Q$ is asymptotically equivalent to the test statistic of the null hypothesis $\alpha=0$ in (3).

${ }^{13}$ See section II of Abel and Mishkin (1983) for a discussion of this point. 
rejection of the null hypothesis is a rejection of market efficiency.

\section{An Empirical Example}

An empirical example from Mishkin (1981b) will illustrate how the tests of market efficiency discussed in the previous section can be conducted. Using bond price data, we conduct a test of the rationality of short-term interest rate forecasts similar to the test with survey data by Friedman (1980). ${ }^{14}$ Consider the following efficient markets model:

$$
\begin{aligned}
& r_{t}=\gamma_{0}+\sum_{i=1}^{6} \gamma_{i} r_{t-i}+u_{t} \\
& B R E T_{t}-r_{t-1}=d+\beta\left(r_{t}-\gamma_{0}-\sum_{i=1}^{6} \gamma_{i} r_{t-i}\right)+\epsilon_{t}
\end{aligned}
$$

where

$r_{t}=90$ day Treasury bill rate at a quarterly rate $B R E T_{t}=$ quarterly return from holding a long-term bond.

The equilibrium return, $E_{m}\left(y_{t} \mid \phi_{t-1}\right)$, is assumed to equal the expected return on a 90 day bill (which is $r_{t-1}$, the bill rate at the end of the quarter $t-1$ ), plus a constant liquidity premium, $d$. Note that in terms of the notation of the previous section, $r_{t}$ corresponds to $X_{t}$, the lagged $r$ 's to $Z$, and the BRET $-r_{t-1}-d$ to $y_{t}$.

We estimate the constrained system (17) for the 1969:3 to $1976: 4$ sample period using non-linear least squares and the weighting procedures outlined above. The results are presented in table $1 .{ }^{15}$ The coefficient on the unanticipated movements of the bill rate is significantly different from zero at the $1 \%$ level, thus indicating that movements in short-term interest rates embody information relevant to the pricing of long-term bonds. Also, as might be expected from the expectations hypothesis of the term structure, the sign of this coefficient is negative, indicating that an unanticipated rise in the bill rate is accompanied by higher long-term rates with a resulting lower bond return. Furthermore, the magnitude of this coefficient is quite close to that found in another study (Mishkin (1978)). ${ }^{16}$

\footnotetext{
14 The reader is referred to Mishkin (1981b) for more details on the model of market equilibrium used here and the data and the motivation behind the particular specification chosen.

15 Note that the results here are slightly different from Mishkin (1981b) because in that paper an iterative procedure was used to weight each equation. Note, however, that under the null hypothesis, either procedure will lead to the same results asymptotically.

${ }^{16}$ Note that in Mishkin (1978) the Treasury bill rates are at an annual rate. Thus an equivalent coefficient on unanticipated movements in the bill rate there should be $1 / 4$ of that found here.
}

TABLE 1. - ESTIMATES OF (17), (18) AND (19):

\begin{tabular}{|c|c|c|c|}
\hline Coefficient of & (17) & (18) & (19) \\
\hline$d$ & $\begin{array}{c}0.00555 \\
(0.00895)\end{array}$ & $\begin{array}{r}-0.01317 \\
(0.06658)\end{array}$ & $\begin{array}{r}-0.01317 \\
(0.06584)\end{array}$ \\
\hline$\beta$ & $\begin{array}{r}-13.59152 \\
(4.48008)\end{array}$ & $\begin{array}{r}-11.3448 \\
\quad(4.60952)\end{array}$ & \\
\hline$\gamma_{0}$ & $\begin{array}{c}0.00597 \\
(0.00229)\end{array}$ & $\begin{array}{c}0.00635 \\
(0.00270)\end{array}$ & \\
\hline$\gamma_{1}$ & $\begin{array}{c}0.61405 \\
(0.17763)\end{array}$ & $\begin{array}{c}0.63039 \\
(0.21038)\end{array}$ & \\
\hline$\gamma_{2}$ & $\begin{array}{c}0.05933 \\
(0.19414)\end{array}$ & $\begin{array}{c}0.10270 \\
(0.22991)\end{array}$ & \\
\hline$\gamma_{3}$ & $\begin{array}{c}0.30926 \\
(0.18974)\end{array}$ & $\begin{array}{c}0.37254 \\
(0.22464)\end{array}$ & \\
\hline$\gamma_{4}$ & $\begin{array}{c}-0.14028 \\
(0.19003)\end{array}$ & $\begin{array}{c}-0.16982 \\
(0.22506)\end{array}$ & \\
\hline$\gamma_{5}$ & $\begin{array}{c}-0.30026 \\
(0.19417)\end{array}$ & $\begin{array}{c}-0.48402 \\
(0.22912)\end{array}$ & \\
\hline$\gamma_{6}$ & $\begin{array}{c}0.03941 \\
(0.18172)\end{array}$ & $\begin{array}{c}0.10476 \\
(0.21513)\end{array}$ & \\
\hline$\theta_{1}$ & & $\begin{array}{c}-0.19925 \\
(1.29565)\end{array}$ & $\begin{array}{c}-0.19925 \\
(1.28144)\end{array}$ \\
\hline$\theta_{2}$ & & $\begin{array}{c}-0.52883 \\
(1.41590)\end{array}$ & $\begin{array}{r}-0.52887 \\
(1.40037)\end{array}$ \\
\hline$\theta_{3}$ & & $\begin{array}{r}-0.77144 \\
(1.38345)\end{array}$ & $\begin{array}{r}-0.77143 \\
(1.36828)\end{array}$ \\
\hline$\theta_{4}$ & & $\begin{array}{c}0.36015 \\
(1.38606)\end{array}$ & $\begin{array}{c}0.36014 \\
(1.37086)\end{array}$ \\
\hline$\theta_{5}$ & & $\begin{array}{c}2.24024 \\
(1.41103)\end{array}$ & $\begin{array}{c}2.24024 \\
(1.39555)\end{array}$ \\
\hline$\theta_{6}$ & & $\begin{array}{c}-0.79670 \\
(1.32488)\end{array}$ & $\begin{array}{r}-0.79670 \\
(1.31035)\end{array}$ \\
\hline
\end{tabular}
1969:3 TO 1976:4 Sample Period

Note: Asymptotic standard errors are in parentheses for (17) and (18); standard errors are in parentheses for (19).

Also found in table 1 are estimates of the unconstrained system ${ }^{17}$

$$
\begin{aligned}
& r_{t}=\gamma_{0}+\sum_{i=1}^{6} \gamma_{i} r_{t-i}+u_{t} \\
& B R E T_{t}-r_{t-1}= d+\beta\left(r_{t}-\gamma_{0}-\sum_{i=1}^{6} \gamma_{i} r_{t-i}\right) \\
&+\sum_{i=1}^{6} \theta_{i} r_{t-i}+\epsilon_{t}
\end{aligned}
$$

and the usual regression equation test for market

\footnotetext{
${ }^{17}$ Note that the liquidity premium, $d$, is estimated in (18) and (19), and thus there are only six constraints implied by rationality and hence only six $\theta$ parameters.
} 
efficiency

$$
B R E T_{t}-r_{t-1}=d+\sum_{i=1}^{6} \theta_{i} r_{t-i}+\epsilon_{t} .
$$

The theory of the previous section indicates that the $\hat{\theta}$ coefficient estimates should be equal for (18) and (19), and the results in table 1 illustrate this conclusion. Even though quite different computer programs (SAS and ESP) were used in estimating (18) and (19), the $\hat{\theta}$ coefficients were equal for at least four significant digits and often more. In addition, the variance-covariance matrix for $\hat{\theta}$ in (18) should equal $[(n-h)(k+1)] /[(k$ $+1)(n-h)-k]=46 / 45$ times the variance-covariance matrix for $\hat{\theta}$ in (19). This is also borne out by the results in table 1 , for the standard errors of the $\hat{\theta}$ in (18) are $(46 / 45)^{1 / 2}$ times the standard errors of the $\hat{\theta}$ in (19).

Three test statistics for rationality and market efficiency are reported in table 2 along with their marginal significance levels: i.e., the probability of obtaining that value of the test statistic or a higher value under the null hypothesis that $\theta=0$. The Wald test statistic was derived from the variance-covariance matrix of (18), the likelihood ratio statistic from the sum of squared residuals of (17) and (18), and the $F$-statistic from the estimates of (19). Note that all three of these statistics yield similar results as would be expected. None leads to rejection of market efficiency, and indeed the marginal significance levels are quite high. The discussion in the previous section also indicates that the Wald statistic should be $[h(n-h)(k+1)] /[(k+1)(n-h)-k)]=$ $6 \times(46 / 45)$ times the $F$-statistic derived from (19), which is what we find in table $2 .^{18}$

\section{Summary and Conclusions}

In this paper we have explored the econometric properties of a test of cross-equation restrictions developed from the theory of efficient markets. We proved the asymptotic equivalence of this test with the common test of market efficiency which tests for the correlation of $y_{t}$ with past information, $Z_{t-1}$. This result is of interest because it is useful for other applications, as in Abel and Mishkin (1983), and because it demonstrates that the cross-equation test can be used for inference under quite general conditions: i.e., regardless of the true value of $\sigma_{u \epsilon}$ and regardless of which past variables are included in $Z$. We also discuss the need for additional restrictions in order to achieve identification. Finally, the empirical application of the cross-equation test illustrates the results derived in the paper.

${ }^{18}$ However, the marginal significance levels are not equal because one is calculated from the $F$-distribution, while the other is calculated from the chi-squared distribution. Of course, this difference disappears asymptotically.
TABle 2. - Tests OF THE NUll Hypothesis $\theta=0$

\begin{tabular}{lccc}
\hline \hline & $\begin{array}{c}\text { Wald Statistic } \\
\text { from (18) }\end{array}$ & $\begin{array}{c}\text { Likelihood Ratio } \\
\text { Statistic from } \\
(17) \text { and (18) }\end{array}$ & $\begin{array}{c}F \text {-Statistic } \\
\text { from (19) }\end{array}$ \\
\hline $\begin{array}{l}\text { Marginal } \\
\text { Significance } \\
\text { Level }\end{array}$ & $\chi^{2}(6)=5.635$ & $\chi^{2}(6)=6.795$ & $F(6,23)=.960$ \\
\hline
\end{tabular}

Note: Marginal Significance Level $=$ the probability of finding that value of the test statistic or higher under the null hypothesis.

Note that both the Wald and likelihood ratio statistics are distributed only asymptotically as $\chi^{2}(6)$

\section{REFERENCES}

Abel, Andrew, and Frederic S. Mishkin, "An Integrated View of Tests of Rationality, Market Efficiency and the ShortRun Neutrality of Monetary Policy," forthcoming in Journal of Monetary Economics (1983).

Barro, Robert, J., "Unanticipated Money Growth and Unemployment in the United States," American Economic Review 67 (Mar. 1977), 101-115.

, "Unanticipated Money, Output, and the Price Level in the United States," Journal of Political Economy 86 (Aug. 1978), 549-580.

Fama, Eugene F., Foundations of Finance (New York: Basic Books, 1976).

Friedman, Benjamin M., "Survey Evidence on the 'Rationality' of Interest Rate Expectations," Journal of Monetary. Economics 6 (Oct. 1980), 453-466.

Granger, Clive W. J., "Investigating Causal Relations by Econometric Models and Cross-spectral Methods," Econometrica 37 (July 1969), 424-438.

Jones, David S., and V. Vance Roley, "Rational Expectations, the Expectations Hypothesis and Treasury Bill Yields: An Econometric Analysis" (Oct. 1981), mimeo, Federal Reserve Bank of Kansas City.

Lucas, Robert E., "Economic Policy Evaluation: A Critique," in Karl Brunner and Allan Meltzer (eds), The Phillips Curve and Labor Markets, Carnegie-Rochester Conference Series on Public Policy, Vol. 1 (Amsterdam: North-Holland Publishing Company, 1976), 19-46.

Mishkin, Frederic S., "Efficient-Markets Theory: Implications for Monetary Policy," Brookings Papers on Economic Activity (3:1978), 707-752.

, "Monetary Policy and Long-Term Interest Rates: An Efficient Markets Approach," Journal of Monetary Economics 7 (Jan. 1981a), 29-56.

, "Are Market Forecasts Rational? American Economic Review 71 (June 1981b), 295-306.

" Monetary Policy and Short-Term Interest Rates: An Efficient Markets-Rational Expectations Approach," Journal of Finance 37 (Mar. 1982), 63-72.

, A Rational Expectations Approach to Macroeconometrics: Testing Policy Ineffectiveness and Efficient Markets Models (Chicago: University of Chicago Press, 1983).

Plosser, Charles I., "The Effects of Government Financing Decisions on Asset Returns," Journal of Monetary Economics 9 (May 1982), 325-352.

Poole, William, "Rational Expectations in the Macro Model," Brookings Papers on Economic Activity (2:1976), 463-505.

Sargent, Thomas J., "Rational Expectations, Econometric Exogeneity, and Consumption," Journal of Political Econ- 
omy 86 (Aug. 1978), 673-700.

"A Note on Maximum Likelihood Estimation of the Rational Expectations Model of the Term Structure," Journal of Monetary Economics 5 (1979), 133-143.

Sargent, Thomas J., and Neil Wallace, "Rational Expectations, the Optimal Monetary Instrument, and the Optimal
Money Supply Rule," Journal of Political Economy 83 (Apr. 1975), 241-254.

Zellner, Arnold, "An Efficient Method of Estimating Seemingly Unrelated Regressions and Tests for Aggregation Bias," Journal of the American Statistical Association 57 (1962), $348-368$.

\title{
THE EMBODIMENT HYPOTHESIS: AN INTERREGIONAL TEST
}

\author{
Richard McHugh and Julia Lane*
}

\section{Introduction}

Several decades ago, Solow (1962) hypothesized that increases in worker productivity could be explained by improvements in the design of new equipment. Output per man-hour would depend not simply on improvements in operations design or the skill level of workers and other factors which would enhance the productivity of all units of capital, and not simply on the level of capital per worker, but would also be dependent on the vintage or age distribution of capital.

Despite the intuitive appeal of this embodiment hypothesis, proponents of embodiment have not found much empirical support in the economics literature on productivity change. In a recent article in this Review, for example, J. K. You (1976) tested for embodiment effects in U.S. manufacturing over the time period from 1929 to 1968 and concluded that technical progress appeared to be disembodied. Earlier, Wickens (1970) used an alternative econometric procedure on U.S. data for 1900 to 1960 to reach essentially the same conclusion: embodiment was not an important factor in the growth of labor productivity.

One thing that is common to tests of the embodiment hypothesis is the use of pure time-series data. In this paper, a report is made on a test of the embodiment hypothesis which utilizes information across regions of the United States.

If technological change is embodied, it is more likely to be uncovered in a cross-section, time-series study. As Denison (1964) notes, for the nation's economy as a whole, the average age of capital changes only very slightly over time. As a result, true embodiment effects may be statistically swamped by other, secular factors.

Received for publication July 27, 1981. Revision accepted for publication September 3, 1982.

* University of Missouri-Columbia and Western Illinois University, respectively.

The authors would like to acknowledge the assistance of Stan Johnson, Richard Green and William Thompson, as well as a referee of this Review. Financial support for this project was provided by the University of Missouri Office of Research.
Given the relatively large variance in the rates of economic growth and capital accumulation over regions of the United States in the past few decades, ages of capital can vary much more substantially among regions than for the aggregation of these regions. The emergence of an industrial base in the South has led to striking differences in the age of capital between these growth areas, and the older industrial Northeast and Midwest. This shift in the location of manufacturing activity, while not greatly disturbing the overall age distribution of capital in the United States, can cause concurrent striking differences in the change in ages of the capital stock among these regions. If this disparity in ages is statistically associated with differences in the rate of growth in output per man-hour, this could be taken as evidence of embodiment.

In this study, data on capital stocks by U.S. Census region for the years 1960 to 1972 have been used to test for embodiment.

\section{Method}

To test for embodiment effects, the same basic procedures as those developed by You (1976) are employed. Total potential output at time $t$ can be defined to equal

$$
P(t)=\int_{-\infty}^{t} P(v, t) d v
$$

where $P(v, t)$ is the potential output which could be produced at time $t$ using

$K(v, t)$, the capital of vintage $v$ surviving at time $t$, and

$L(v, t)$, labor working with $K(v, t)$.

Assuming a Cobb-Douglas production function with constant returns to scale,

$$
P(v, t)=A e^{\gamma t}\left(e^{\lambda t} K(v, t)\right)^{\alpha} L(v, t)^{1-\alpha}
$$

where

$\gamma$ is the rate of disembodied technological change

$\lambda$ is the rate of embodied technological change

$\boldsymbol{\alpha}$ is the capital-output elasticity. 